\title{
Formação Científica e sua Relação com a Capacitação Profissional do Psicólogo
}

\author{
Valquiria Maria Gonçalves ${ }^{1}$ \\ Nádia Kienen \\ Verônica Bender Haydu \\ Programa de Mestrado em Análise do Comportamento e Departamento de Psicologia Geral \\ e Análise do Comportamento da Universidade Estadual de Londrina, Londrina, PR, Brasil
}

\begin{abstract}
Resumo
Uma revisão bibliográfica foi realizada para identificar as contribuições da formação científica para a capacitação profissional do psicólogo. Em bases de dados científicas, foram selecionados 14 trabalhos sobre formação científica do psicólogo e sua capacitação profissional. A partir da identificação de características como natureza do trabalho, objetivos, método e contribuições apresentadas, foram formuladas as categorias para análise dos resultados, sendo essas: (a) concepção sobre pesquisa, formação científica e participação em atividade de pesquisa; (b) reflexão sobre a importância da formação científica para a capacitação profissional do psicólogo; (c) caracterização de comportamentos profissionais relativos à formação científica do psicólogo; (d) características dos objetivos de ensino presentes nos planos de curso e de ensino de disciplinas relacionadas à capacitação científica na graduação em Psicologia; (e) programas de ensino de comportamentos profissionais que compõem a formação científica do psicólogo. Destaca-se o desconhecimento dos alunos sobre a possibilidade de participação em atividades de pesquisa e a relevância da caracterização de comportamentos científicos/profissionais.
\end{abstract}

Palavras-chave: Formação científica, formação do psicólogo, formação profissional, Programação de Ensino, revisão da bibliografia.

\section{Scientific Training and its Relation with the Professional Training of Psychologist}

\begin{abstract}
A literature review was conducted to identify how scientific training contributes to the professional training of psychologists. In scientific databases, fourteen papers relating to scientific training of psychologists as part of their professional training during their degree courses were selected. Based on the identification of characteristics such as their nature, aims, methods and findings, categories were formulated in order to analyze the results of the studies, namely: (a) conception of research, scientific training and participation in research activity; (b) reflection on the importance of scientific training for the professional training of psychologists; (c) characterization of professional behaviors related to the scientific training of psychologists; (d) characteristics of teaching goals present in course plans and
\end{abstract}

Endereço para correspondência: Universidade Estadual de Londrina, Centro de Ciências Biológicas, Departamento de Psicologia Geral e Análise do Comportamento, Rodovia Celso Garcia Cid, PR 445, Km 380, Campus Universitário, Caixa Postal 10.011, Londrina, PR, Brasil 86057-970. E-mail: valk.mg@gmail.com, nadiakienen@gmail.com e veronicahaydu@gmail.com

Agências de financiamento: Coordenação de Aperfeiçoamento de Pessoal de Nível Superior (CAPES) e Fundação Araucária. 
teaching plans for scientific training as part of Psychology degree courses; (e) teaching programs to develop professional behaviors as part of the scientific training of psychologists. A noteworthy finding is students' unawareness about the possibility of participating in research activities and the relevance of the characterization of scientific and professional behaviors.

Keywords: Scientific training, psychologists' formation, professional training, Teaching Programming, literature review.

\section{Formación Científica y su Relación con la Capacitación Profesional del Psicólogo}

\section{Resumen}

Una revisión bibliográfica fue realizada para identificar las contribuciones de la formación científica en la capacitación profesional del psicólogo. En bases de dados científicas, fueron seleccionados 14 trabajos sobre la formación científica del psicólogo y su capacitación profesional. A partir de la identificación de categorías como naturaleza del trabajo, objetivos, método y las contribuciones realizadas, fueron formuladas las categorías para el análisis de los resultados, siendo ellas: (a) concepción al respecto de la investigación, formación científica y participación en actividades de investigación; (b) reflexión sobre la importancia de la formación científica para la formación profesional del psicólogo; (c) caracterización de la conducta profesional con respecto a la formación científica del psicólogo; (d) características de los objetivos de la enseñanza presentes en el programa del curso y de la enseñanza de materias relacionadas con la formación científica en Psicología; (e) programas de enseñanza de conductas profesionales que componen la formación científica del psicólogo. Destacarse la ignorancia de los estudiantes acerca de la posibilidad de participar en actividades de investigación y la relevancia de la caracterización de las conductas científicas/profesionales.

Palabras clave: Formación científica, formación del psicólogo, formación profesional, programación de la educación, revisión de la literatura.

Uma das áreas para a qual o profissional de Psicologia deve ser capacitado a atuar durante o curso de graduação é a área científica. Essa capacitação requer o desenvolvimento de comportamentos como observar os fenômenos comportamentais, formular questões, buscar bibliografia, avaliar qual o melhor método de intervenção e de investigação, verificar e relatar os resultados, dentre outros. O desenvolvimento dessas habilidades é fundamental para que os profissionais dessa área sejam capazes de produzir conhecimento e transformá-lo em tecnologia, o que é considerado um fator essencial não apenas à formação de qualquer profissional, mas também ao desenvolvimento de uma nação (Viecili, 2008).

Desde a regulamentação da profissão de psicólogo no Brasil, em 1962, e após anos de discussões sobre o papel e importância da formação científica na graduação em Psicologia, o espaço dessa formação tem sido garantido em termos legais por meio das Diretrizes Curriculares Nacionais para os cursos de graduação em Psicologia, por meio da Resolução $\mathrm{n}^{0} 5$, de 15 de março de 2011 (Ministério da Educação, Conselho Nacional de Educação/Câmara da Educação Superior, 2011). Essas diretrizes, além de apresentarem como "meta central a formação do psicólogo voltada para a atuação profissional, para a pesquisa e para o ensino de Psicologia" (Art. $3^{\circ}$ ), destacam que, para que esse objetivo seja alcançado, devem ser observados princípios e compromissos como a "Construção e desenvolvimento do conhecimento científico em Psicologia", além de outros, muitos dos quais relacionados à capacitação científica. Entretanto, psicólogos, pesquisadores e professores de Psicologia têm discutido, em encontros científicos e da categoria profissional, a defasagem entre teoria, pesquisa e 
intervenção na formação e prática do psicólogo, que resultam em problemas relacionados à baixa qualidade da produção científica e ao uso de práticas ineficazes para o atendimento das diversas demandas sociais (Cruces, 2008).

Evidências dessa defasagem foram assinaladas por Bariani (1995) e Silva (2007), que apontam que profissionais graduados em diferentes cursos, ao ingressarem em cursos de pós-graduação, apresentam dificuldades, observando-se, em determinados casos, ausência de domínio de conhecimentos de metodologia científica relevantes para o desenvolvimento de seus projetos e para a produção de conhecimento. $\mathrm{O}$ resultado são trabalhos que apresentam falhas prejudiciais à qualidade do que foi produzido (Bariani, 1995; Silva, 2007), indicando que o ensino não foi capaz de garantir o desenvolvimento de comportamentos científicos.

Mesmo na pós-graduação, a meta de formação científica pode não estar sendo cumprida. Exemplo disso foi verificado por Silva (2007) que, a partir da revisão de dissertações de alunos de um programa de pós-graduação em Psicologia, examinou a classe geral de comportamentos "delimitar problema de pesquisa", constatando que a maioria dos autores das dissertações não enunciou de forma clara o problema de pesquisa. A autora concluiu que provavelmente os comportamentos relativos a "delimitar problema de pesquisa" não são ensinados a esses alunos.

Uma revisão dos estudos em Psicologia a respeito da formação científica e profissional pode fornecer subsídios para discutir a capacitação do psicólogo. No que se refere à formação em pesquisa, pode-se buscar identificar como se apresentam e caracterizam as deficiências presentes no ensino de comportamentos relativos à pesquisa durante a graduação, bem como identificar alternativas para tais deficiências. Com base no exposto, o presente estudo teve por objetivo avaliar as contribuições da formação científica durante a graduação para a capacitação profissional do psicólogo por meio de revisão da bibliografia pertinente ao tema. Para isso, realizou-se um exame de artigos científicos, dissertações e teses em Psicologia que apresentam resultados sobre formação científica e sua relação com a ca- pacitação do futuro psicólogo, durante o curso de graduação.

\section{Método}

O presente estudo consistiu em um estudo teórico realizado a partir do levantamento de trabalhos sobre a temática da formação científica do psicólogo. A seleção do material foi feita a partir de pesquisa bibliográfica em base de dados online por meio do portal da Coordenação de Aperfeiçoamento de Nível Superior (CAPES) disponível em http://periodicos.capes.gov.br, no qual foram acessadas duas bases de dados com conteúdo relacionado à Psicologia em língua portuguesa (IndexPsi e SciELO [Scientific Electronic Library Online]). Também foi realizada busca em dois repositórios brasileiros online de dissertações e teses (Biblioteca Digital de Teses e Dissertações [BDTD] e Repositório de Conteúdo Digital da Universidade Federal de Santa Catarina [UFSC], no acervo do Programa de Pós-graduação em Psicologia [PPGP]).

Com base no tema escolhido foram definidos os seguintes descritores nas bases de dados: formação científica, formação em pesquisa, psicologia, psicólogo, formação do psicólogo, pesquisa psicológica, metodologia científica, metodologia em pesquisa, capacitação científica, capacitação profissional. Foram pesquisados trabalhos que continham tais termos em qualquer parte do texto. Os termos foram pesquisados sozinhos e em busca realizada por meio de operadores booleanos, no modo de "pesquisa avançada" das bases de dados, a fim de refinar os resultados da pesquisa de acordo com o objetivo do trabalho.

A partir da análise dos títulos e resumos dos trabalhos encontrados, foram selecionados os que abordavam o tema da formação científica e sua relação com a capacitação do psicólogo durante a graduação, sendo excluídos todos os que não tinham uma especificação desse tema.

Na busca realizada na BDTD (disponível em http://bdtd.ibict.br), foram usadas as principais palavras-chave relacionadas ao tema, no campo "Resumo" da procura avançada. Foram elas: formação do psicólogo, formação científi- 
ca, formação em pesquisa, capacitação científica e metodologia científica. Do mesmo modo, a partir da análise dos títulos e resumos dos trabalhos encontrados nessa busca, foram selecionados os que se relacionavam com o tema da pesquisa, resultando, assim, em um trabalho recuperado.

Realizou-se ainda busca no Repositório de Conteúdo Digital da UFSC, no acervo do PPGP. Tal escolha foi feita considerando que uma das linhas de pesquisa do PPGP abrange trabalhos sobre capacitação do psicólogo, incluindo capacitação científica, orientados pelo Prof. Dr. Sílvio Paulo Botomé e pela Profa. Dra. Olga Mitsue Kubo. Destaca-se que o trabalho que foi recuperado na BDTD fazia parte do PPGP da UFSC.

Em uma primeira fase foram realizadas leituras dos trabalhos ou de partes deles para identificação dos aspectos e informações apresentadas em cada um, sendo estas: tema, objetivo, tipo de pesquisa (se teórica, empírica, etc.), natureza do trabalho (se artigo, tese, dissertação), método utilizado e discussões e conclusões apresentadas no trabalho.

Posteriormente, foram propostas categorias para análise dos trabalhos constituintes da amos- tra, sendo formuladas considerando os objetivos de cada trabalho e as considerações feitas neles, agrupando-os de acordo com as semelhanças apresentadas nesse sentido. As categorias de análise formuladas foram: (a) concepção sobre pesquisa, formação científica e participação em atividade de pesquisa; (b) reflexão sobre a importância da formação científica para a capacitação profissional do psicólogo; (c) caracterização de comportamentos profissionais relativos à formação científica do psicólogo; (d) características dos objetivos de ensino presentes nos planos de curso e de ensino de disciplinas relacionadas à capacitação científica na graduação em Psicologia e (e) programas de ensino de comportamentos profissionais que compõem a formação científica do psicólogo.

\section{Resultados e Discussão}

A quantidade de artigos inicialmente encontrada, selecionada e recuperada na busca realizada com cada descritor pesquisado está distribuída na Tabela 1. Pode-se observar que apenas os descritores "Formação em pesquisa" e "Formação do psicólogo", e as combinações "Formação

Tabela 1

Descritores Pesquisados nas Bases de Dados e a Quantidade Correspondente de Artigos Inicialmente Encontrados, Selecionados e Recuperados para Análise

\begin{tabular}{cccc}
\hline Descritores pesquisados nas bases de dados & $\begin{array}{c}\text { Qtde de trabalhos } \\
\text { encontrados }\end{array}$ & $\begin{array}{c}\text { Qtde de trabalhos } \\
\text { selecionados }\end{array}$ & $\begin{array}{c}\text { Qtde de trabalhos } \\
\text { recuperados }\end{array}$ \\
\hline Formação científica & 124 & 6 & 0 \\
Formação em pesquisa & 137 & 8 & 2 \\
Formação do psicólogo & 39 & 4 & 0 \\
Pesquisa psicológica & 25 & 0 & 0 \\
Capacitação científica & 41 & 0 & 0 \\
Capacitação Profissional & 38 & 0 & 1 \\
Formação científica $A N D$ Psicólogo & 19 & 4 & 1 \\
Formação em pesquisa $A N D$ Psicólogo & 14 & 2 & 1 \\
Metodologia científica $A N D$ Psicologia & 37 & 3 & 0 \\
Metodologia de pesquisa $A N D$ Psicologia & 32 & 2 & 0 \\
Capacitação científica $A N D$ Psicólogo & 1 & 0 & 1 \\
Capacitação profissional $A N D$ Psicólogo & 2 & & \\
\hline
\end{tabular}


científica $A N D$ Psicólogo", "Formação em pesquisa $A N D$ Psicólogo", "Metodologia científica $A N D$ Psicologia" e "Metodologia de pesquisa $A N D$ Psicologia" produziram trabalhos a serem recuperados, cujo processo de seleção encontra-se no fluxograma representado na Figura 1. Ve- rifica-se nessa figura que dos 698 trabalhos resultantes da busca inicial, foram recuperados 47 trabalhos completos, sendo 33 repetidos, permanecendo 14 trabalhos para compor a amostra de análise desse estudo (oito artigos, quatro dissertações de mestrado e duas teses de doutorado).
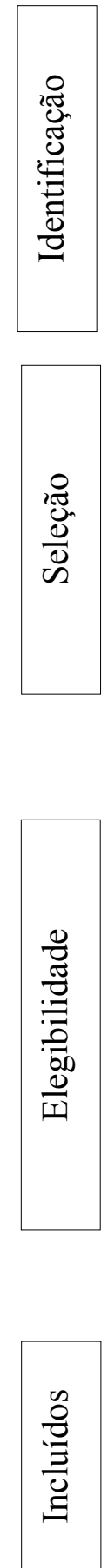

Trabalhos encontrados nas bases de dados $(n=509)$

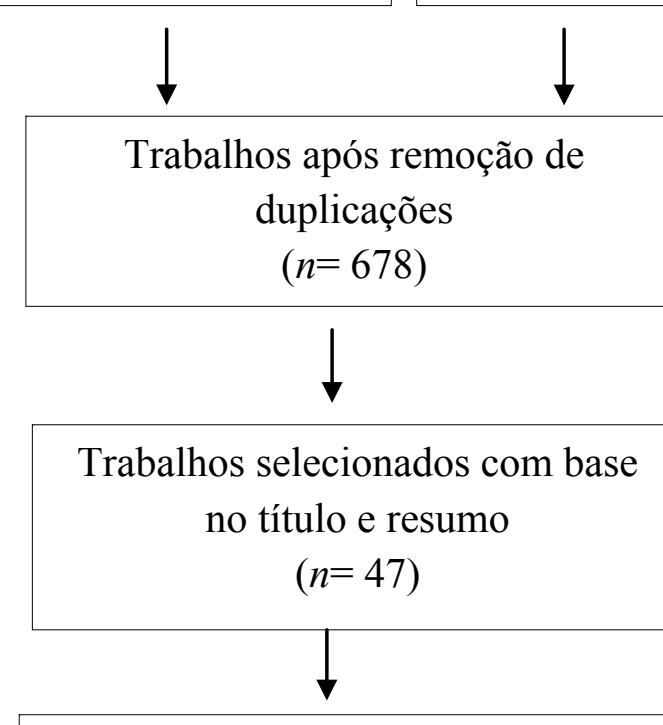

Trabalhos completos recuperados $(n=47)$

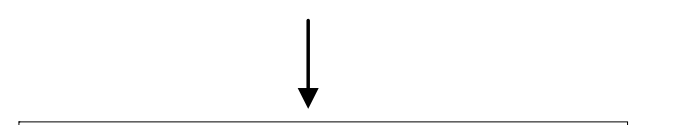

Trabalhos excluídos que não atingiram o critério a partir da leitura do texto completo

$$
(n=33)
$$

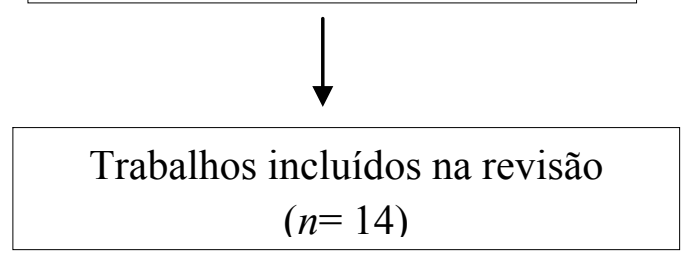

Figura 1. Fluxograma da pesquisa e quantidade de trabalhos localizados, selecionados e recuperados.

A partir da leitura dos trabalhos, esses foram classificados em cinco categorias, de acordo com a abordagem que faziam sobre a temática da contribuição da formação científica para a capacitação profissional do psicólogo. Para tal classificação, foi considerado principalmente $o$ 
objetivo central de cada trabalho, além dos resultados e conclusões apresentados neles. Na Tabela 2 são explicitadas as categorias e as referên- cias dos trabalhos que compõem cada uma delas. A seguir, apresenta-se a descrição e análise dos estudos com base nas categorias formuladas.

Tabela 2

Artigos Selecionados nas Bases de Dados e Distribuição de Acordo com as Categorias Formuladas para Classificação e Análise

\begin{tabular}{|c|c|c|c|}
\hline Número & Categorias & Referência do Trabalho & Tipo de publicação \\
\hline \multirow{5}{*}{1} & \multirow{5}{*}{$\begin{array}{l}\text { Concepção sobre pesquisa, } \\
\text { formação científica } \\
\text { e participação } \\
\text { em atividade } \\
\text { de pesquisa }\end{array}$} & Bariani (1995) & Artigo \\
\hline & & Bastian et al. (2000) & Artigo \\
\hline & & Guareschi, Wendt, \& Dhein (2011) & Artigo \\
\hline & & Leme (2001) & Artigo \\
\hline & & Seidl-de-Moura, Bosco, Diniz, \& Santos (1993) & Artigo \\
\hline \multirow{3}{*}{2} & \multirow{3}{*}{$\begin{array}{c}\text { Reflexão sobre a importância } \\
\text { da formação científica } \\
\text { para a capacitação } \\
\text { do profissional psicólogo }\end{array}$} & Ades (1981) & Artigo \\
\hline & & Cruces (2008) & Artigo \\
\hline & & Weber (1985) & Artigo \\
\hline \multirow{4}{*}{3} & \multirow{4}{*}{$\begin{array}{c}\text { Caracterização } \\
\text { de comportamentos } \\
\text { profissionais que compõem } \\
\text { a formação científica } \\
\text { do psicólogo }\end{array}$} & Goecks (2011) & Dissertação \\
\hline & & Luca (2008) & Dissertação \\
\hline & & Noceti (2011) & Dissertação \\
\hline & & Viecili (2008) & Tese \\
\hline 4 & $\begin{array}{l}\text { Características dos objetivos } \\
\text { de ensino presentes nos planos } \\
\text { de curso e de ensino } \\
\text { de disciplinas relacionadas } \\
\text { à capacitação científica } \\
\text { na graduação em psicologia }\end{array}$ & Grando (2009) & Dissertação \\
\hline 5 & $\begin{array}{l}\text { Programas de ensino de } \\
\text { comportamentos profissionais } \\
\text { que compõem a formação } \\
\text { científica do psicólogo }\end{array}$ & Luca (2013) & Tese \\
\hline
\end{tabular}

Concepção sobre Pesquisa, Formação Científica e Participação em Atividade de Pesquisa

Dos 14 trabalhos analisados, cinco foram enquadrados na categoria denominada de "Concepção sobre pesquisa, formação científica e participação em atividade de pesquisa", conforme apresentado na Tabela 1. Nos estudos de Bariani (1995), Bastian et al. (2000) e Seidl-de-Moura et al. (1993), foram aplicados questionários a estudantes do curso de Psicologia para, de modo geral, investigar o conhecimento e a concepção que eles têm sobre formação científica e sobre a participação deles em atividades de pesquisa. $\mathrm{O}$ estudo de Bariani (1995) foi realizado com 43 estudantes de Psicologia que realizavam atividades de pesquisa, enquanto que os outros dois estudos (Bastian et al., 2000; Seidl-de-Moura et al., 1993) foram realizados, respectivamente, com 82 e 93 estudantes participantes ou não de atividades de pesquisa.

Em Leme (2001), foi utilizada uma coleta de depoimentos de alunos de iniciação científica e de professores com a finalidade de investigar a 
contribuição da formação em pesquisa, por meio de atividades de iniciação científica (IC), para a formação do psicólogo. No quinto estudo a compor essa categoria (Guareschi et al., 2011), foram analisadas respostas dos estudantes de Psicologia ao questionário socioeconômico do Exame Nacional de Desempenho de Estudantes (ENADE) do ano de 2006. Essas respostas referiam-se a questões sobre participação em atividades de pesquisa/iniciação científica, monitoria e extensão, mas para o presente estudo, foram utilizados somente os dados que se referem à pesquisa/iniciação científica.

Os resultados encontrados apontam que a pesquisa, segundo os estudantes, é considerada importante e que a prática de atividades de pesquisa contribuiria para a formação profissional do psicólogo. No estudo de Seidl-de-Moura et al. (1993), os alunos responderam que consideram que a pesquisa é importante, porque, dentre outros fatores, é "fonte de conhecimentos", possibilita "o progresso da ciência" e "pode ser fonte de mudanças na psicologia ou de mudanças sociais" (p. 27). Além disso, os estudantes destacaram que o papel do pesquisador é importante, "porque é ele o agente, o sujeito nesse processo que permite as conquistas propiciadas pela pesquisa" (p. 27), mostrando uma visão idealizada e distante da prática na graduação. Os resultados apresentados por Bariani (1995) indicam que os alunos-pesquisadores consideravam que a participação em atividade de pesquisa era de grande importância para a vida acadêmica e para a formação profissional.

Nos depoimentos coletados com os alunos de iniciação científica no estudo de Leme (2001), foram mencionados aspectos positivos da participação na atividade de pesquisa, que incluíam o desenvolvimento de uma relação mais flexível com o conhecimento, principalmente por meio do desenvolvimento de um senso mais crítico e de uma mudança do modo de pensar; a promoção da autonomia e de um posicionamento ativo diante do conhecimento e dos problemas científicos; e a relação de trocas estabelecida com o orientador. Por sua vez, Bastian et al. (2000) partiram da premissa de que os psicólogos não valorizam a pesquisa científica, dedicando-se mais a aspectos técnicos da profissão, porém, $99 \%$ dos estudantes responderam que consideravam que a pesquisa fazia parte das atividades do psicólogo.

Outro tópico encontrado nos estudos foi o conhecimento sobre pesquisa e o interesse que os estudantes tinham em realizar esse tipo de atividade, sendo constatado por Bastian et al. (2000) e Seidl-de-Moura et al. (1993) que os alunos apresentavam baixa frequência desses comportamentos. Esses resultados, no entanto, podem estar relacionados a outro fator, que foi mencionado em todos os trabalhos dessa categoria: a pouca ou até mesmo a ausência de divulgação das atividades de pesquisa aos estudantes. Bastian et al. (2000) apresentam que $60 \%$ da amostra do estudo $(n=82)$ não sabia da possibilidade de os alunos de graduação realizarem atividades de pesquisa e alguns alunos-pesquisadores responderam que a pesquisa é uma "atividade trabalhosa", "difícil" e até mesmo "chata", o que pode contribuir também com o desinteresse dos alunos em relação à prática de pesquisa.

A falta de interesse e de conhecimento sobre pesquisa e sobre oportunidades de realização desse tipo de atividade resulta em pouca participação, como foi evidenciado pelo estudo de Seidl-de-Moura et al. (1993), em que apenas $10 \%$ dos estudantes da amostra $(n=93)$ participavam ou haviam participado de atividades de pesquisa. No estudo de Guareschi et al. (2011), desenvolvido em âmbito nacional, $57,1 \%$ dos estudantes de Psicologia $(n=23.613)$ não participavam ou não haviam participado de atividades de pesquisa durante a graduação.

A partir dos resultados encontrados sobre o desconhecimento dos alunos a respeito de pesquisa e das oportunidades de realização desse tipo de atividade, Bastian et al. (2000) ampliaram o estudo com a realização de grupos e de intervenção com os estudantes que participaram de sua pesquisa inicial, sendo o único trabalho dessa categoria em que foi realizada uma intervenção. Seu objetivo foi a devolução de resultados, bem como fornecer informações e esclarecimentos sobre as atividades de pesquisa oferecidas pela universidade em que estudavam, para incentivar a participação dos estudantes em projetos de pesquisa. 
Sobre a relação da formação científica durante a graduação e a pós-graduação, nos depoimentos dos professores do estudo de Leme (2001) foi mencionado que a experiência com pesquisa realizada por meio da IC seria um preparo do aluno para a pós-graduação, pois os alunos utilizam o que aprenderam durante a IC no projeto a ser desenvolvido na pós-graduação. No estudo de Guareschi et al. (2011), pelas respostas dos estudantes, quando examinada a soma dos índices de contribuição plena, parcial e restrita da IC à formação profissional, em geral, a realização da atividade é considerada de maior contribuição para a formação por estudantes de instituições de ensino superior (IES) com programas de pós-graduação (PPG). É provável que tal fato seja devido a um maior incentivo por parte dos docentes para que os alunos tenham contato com práticas de pesquisa e, assim, estejam mais preparados para os cursos de pós-graduação da instituição.

Ainda no estudo de Guareschi et al. (2011), foram relacionadas as respostas sobre participação dos estudantes em atividades de pesquisa e os índices de contribuição dessa atividade à formação profissional. Verificou-se que o índice de estudantes que participaram ou participavam de atividades de IC e consideravam que tal experiência contribuía plenamente à formação profissional era inferior ao índice de estudantes que não participavam ou haviam participado de atividades de pesquisa na graduação. É importante destacar que no estudo de Leme (2001), os alunos apontaram como pontos negativos da IC os diferentes estilos de orientação dos professores, sendo que alguns tratavam o aluno como um simples realizador de tarefas, não possibilitando muita autonomia, enquanto outros professores proporcionavam uma orientação considerada muito "solta", sem diretrizes, deixando o aluno sem saber como proceder. Outro ponto negativo citado pelos alunos com relação à IC foi uma especialização precoce devido ao trabalho com objetos de estudo muito específicos, que pode não ser vantajoso ao estudante na graduação.

Relacionado ainda com a participação em atividades de pesquisa, porém mais especificamente ao motivo da procura por esse tipo de atividade, no estudo de Bariani (1995), os estudantes, de forma geral, responderam que a busca por projetos de pesquisa era por curiosidade em saber mais sobre pesquisa em Psicologia e por interesse em aprender mais sobre metodologia de pesquisa. A maioria desses alunos respondeu ter um conhecimento apenas razoável em metodologia de pesquisa. Esses resultados podem ter relação com o que Seidl-de-Moura et al. (1993) discutiram ao constatarem um panorama de insatisfação dos alunos com o ensino de metodologia de pesquisa e a formação científica durante a graduação, cujas respostas referiam-se a um baixo número de disciplinas sobre pesquisa. Além disso, de acordo com as autoras, as disciplinas de metodologia científica pareciam não atender aos objetivos a que se propunham.

Um dos compromissos da Educação Superior, segundo Bariani (1995), seria oferecer a formação científica como parte importante da capacitação do profissional. Contudo, parece que esse compromisso não tem sido totalmente cumprido, sendo dada mais ênfase à formação técnica do profissional, em detrimento à formação científica. Isso demonstra uma compreensão dessas formações não como complementares e interdependentes, mas como distintas e também distantes uma da outra. A autora discute ainda que a universidade deveria dar condições para que o aluno desenvolvesse uma "atitude científica", o que seria muito mais do que realizar pesquisas somente, o que também foi apontado nas reflexões dos artigos de Ades (1981) e Cruces (2008)

\section{Reflexão sobre a Importância da Formação Cientifica para a Capacitação Profissional do Psicólogo}

Três artigos selecionados (Ades, 1981; Cruces, 2008 e Weber, 1985) foram distribuídos na categoria "Reflexão sobre a importância da formação científica para a capacitação profissional do psicólogo". Sendo dois artigos da década de 1980 e um da década de 2000, cada um traz reflexões considerando o cenário dos diferentes períodos da história da Psicologia brasileira, no que tange à formação profissional e científica. E embora distantes com relação à 
época em que foram escritos, os artigos acabam por abordar problemas semelhantes, apontando que os problemas encontrados na década de 2000 parecem ser um desenrolar dos apresentados nos anos 1980

Com base em um simpósio realizado durante a VIII Reunião Anual de Psicologia da Sociedade de Psicologia de Ribeirão Preto, no ano de 1978, com o título "Formação em psicologia, o ponto de vista dos profissionais", Ades (1981) escreveu seu artigo de reflexão. Assim, partindo da premissa de que não existiria real motivo para se considerar como antagônicas ou incompatíveis as atividades de pesquisa e de aplicação em Psicologia, bem como considerando que integrar pesquisa e aplicação só traria benefícios à formação do psicólogo, discutiu o "treino em pesquisa na graduação em psicologia como um caminho para a formação de uma atitude criativa de investigação e teorização" (p. 113). Isso, segundo o autor, é válido tanto para a carreira de pesquisador, como para a atuação profissional do psicólogo em outros contextos e atividades.

Dados de estudos desenvolvidos por outros pesquisadores na década de 1970 , tanto no Brasil como no exterior, foram apresentados por Ades (1981), que apontam haver pouco interesse dos estudantes com relação à pesquisa, em detrimento do grande interesse para a área aplicada. Resultado semelhante aos apresentados na categoria "Concepção sobre pesquisa, formação científica e participação em atividade de pesquisa", por Bariani (1995), Bastian et al. (2000), Guareschi et al. (2011), Leme (2001) e Seidl-de-Moura et al. (1993).

A partir das respostas dadas em um questionário destinado a professores de Psicologia antes da VIII Reunião Anual de Psicologia da Sociedade de Psicologia de Ribeirão Preto, no ano de 1978, Ades (1981) apresentou que nas questões relativas à formação em pesquisa, respondida por 29 professores, a maioria relatou que não existia, na instituição em que era docente, prática de pesquisa pelos alunos e que os cursos de metodologia eram insuficientes para o ensino de pesquisa. Aproximadamente $80 \%$ dos professores que responderam a essas questões se manifestaram a favor do treino em pesquisa du- rante o curso de graduação em Psicologia. Uma das respostas dizia que "a não ser que estejamos formando justamente aplicadores de técnicas, o treino em pesquisa é essencial; não, talvez, com o objetivo de formar uma pessoa competente e autossuficiente no planejamento e execução de pesquisas, mas para dar "espírito crítico"" (Ades, 1981, p. 116).

Ainda sobre a capacitação científica e a relação com a falta de treino em pesquisa e a insuficiência dos cursos de metodologia científica, Ades (1981) pontuou que se a intenção for que os estudantes possam aprender muito mais do que reproduzir fragmentos e técnicas, é necessário que haja a participação dos alunos numa prática de pesquisa. A atividade prática possibilita ao estudante apresentar um processo de mudança comportamental facilitado pela demonstração do quanto a pesquisa pode contribuir com a formação profissional.

Uma reflexão sobre o espaço da pesquisa na formação do psicólogo foi feita por Weber (1985) no simpósio "A formação do pesquisador em Psicologia e o curso de graduação - responsabilidades negligenciadas", promovido pela Associação Nacional de Pesquisa e Pós-Graduação em Psicologia, considerando o Currículo Mínimo de Psicologia vigente na época, previsto no artigo 10 do Decreto-lei $n^{\circ}$. 53.464, de 21 de janeiro de 1964. O Currículo Mínimo consistia em algumas disciplinas obrigatórias que os cursos deveriam oferecer e algumas opcionais, escolhidas pelas instituições de nível superior, porém nenhuma dessas disciplinas era relacionada à formação científica especificamente. De acordo com Weber, não seria possível pensar o Currículo Mínimo e o espaço da pesquisa nele sem antes pensar na própria formação profissional do psicólogo. A autora destacou que o Currículo Mínimo privilegiava a profissionalização do psicólogo, principalmente para atuação em clínica. No entanto, ao se pensar a Psicologia como ciência e profissão, novas exigências eram evidentes, não se tratando apenas da inclusão de disciplinas de pesquisa no Currículo Mínimo, mas da necessidade de se analisar como seria realizado o processo de inserção nos grupos de pesquisa e na participação em projetos de pesquisa. Uma 
inserção efetiva contribuiria não somente para a formação de pesquisadores, mas principalmente, para a formação de profissionais capacitados a serem bons consumidores de pesquisa.

A importância da pesquisa na formação do psicólogo também foi destacada por Cruces (2008), apresentando um panorama histórico da preparação desse tipo de profissional desde os tempos iniciais de criação de cursos de Psicologia no Brasil e a regulamentação da profissão em 1962. A autora discutiu que com a expansão dos cursos de Psicologia, iniciou-se um processo de defasagem entre teoria, pesquisa e extensão, sendo a pesquisa colocada em segundo plano ou até mesmo não contemplada durante a formação. Tal fato acarretou problemas na atuação desse profissional, devido a um preparo inadequado nos cursos de formação, que levou ao questionamento sobre a adequação de práticas do psicólogo nem sempre eficazes e condizentes com a realidade, resultando em um movimento de reflexão a respeito dessa situação, principalmente nos anos 1980, o que pode ser observado pelos artigos de Ades (1981) e Weber (1985).

Em seu artigo, Cruces (2008) destacou ainda o importante papel da formação científica para a formação de um bom profissional, papel esse discutido em encontros de associações e grupos tais como da American Psychological Association (APA), Primera Conferencia Latinoamericana sobre Entrenamiento em Psicologia, dentre outros. A preparação em pesquisa seria o modo de capacitar bons profissionais, capazes de avaliar, questionar, observar, levantar hipóteses e propor intervenções eficazes e adequadas às necessidades. No entanto, no Brasil, não havia disciplinas relativas à pesquisa no Currículo Mínimo para o curso de Psicologia, fato esse que só se modificou a partir de 2004 - com discussões sobre essas modificações iniciadas na década de 1980 - a partir das Diretrizes Curriculares Nacionais, que substituía o rol de disciplinas do Currículo Mínimo por competências e habilidades necessárias ao psicólogo, contemplando e garantindo agora a formação científica.

A partir da análise dos três artigos apresentados nessa categoria, pode-se observar que a discussão sobre o espaço e a importância da for- mação em pesquisa para a formação profissional do psicólogo está presente há anos e, embora muitos pontos ainda precisem de mais discussão e, principalmente, de mudanças, houve avanços em termos de políticas públicas para o ensino de Psicologia. Porém, tais avanços precisam ser garantidos por meio das práticas dentro das instituições, envolvendo desde os planos dos cursos de Psicologia, até o modo pelo qual é realizada a capacitação científica e como são ensinadas as disciplinas de metodologia científica. Deve-se analisar, inclusive, o quanto a capacitação em pesquisa que tem sido realizada nas instituições de ensino superior contribui para uma formação profissional de melhor qualidade, tendo em vista que tipo de profissional a universidade deseja formar.

\section{Caracterização de Comportamentos Profissionais que Compõem a Formação Científica do Psicólogo}

Dentre os estudos selecionados, quatro (Goecks, 2011; Luca, 2008; Noceti, 2011; Viecili, 2008) foram categorizados como "Caracterização de comportamentos profissionais que compõem a formação científica do psicólogo". Todos os estudos dessa categoria tinham por objetivo identificar quais classes de comportamentos constituem classes gerais relativas a comportamentos acadêmicos, profissionais e científicos que, no presente artigo, são relacionados à formação científica do psicólogo. Dentre as classes gerais de comportamentos estudadas estão "avaliar a confiabilidade de informações" (Luca, 2008), "delimitar problema de pesquisa a partir de perguntas" (Noceti, 2011), "ler textos acadêmicos" (Goecks, 2011) e classes de comportamentos profissionais que compõem a formação do psicólogo para intervir indiretamente por meio de pesquisa (Viecili, 2008).

A caracterização das classes de comportamentos que compõem uma determinada atuação ou função de alguém na sociedade consiste em uma das várias etapas - e talvez a mais importante - da Programação de Ensino, que é uma tecnologia de ensino produzida a partir da Análise Experimental do Comportamento (Kienen, Kubo, \& Botomé, 2013). De modo geral, entre 
as etapas, tem-se inicialmente a caracterização de necessidades sociais do aprendiz, a partir das quais são identificados que comportamentos necessitam ser aprendidos a fim de que ele possa lidar melhor com essas necessidades. O ensino desses comportamentos é que lhe permitirá transformar a realidade social com a qual se deparará depois de passado o período de ensino. Essa primeira etapa de identificação e caracterização dos comportamentos que constituirão a atuação do aprendiz na sociedade possibilitará o planejamento de várias outras etapas de ensino efetivo desses comportamentos. A partir disso podem ser programadas condições de ensino para que os comportamentos sejam desenvolvidos, sendo então propostas as atividades para tal. A avaliação da eficiência e eficácia dos programas de ensino constitui parte final do processo de Programação de Ensino e possibilita o aperfeiçoamento do programa de ensino desenvolvido.

Os estudos da presente categoria destacam que caracterizar as classes de comportamentos é importante, pois possibilita conhecer o que se deve ensinar para capacitar os futuros profissionais e pesquisadores. Dessa forma, a partir da identificação dos comportamentos constituintes das classes gerais estudadas, é possível que professores formulem objetivos de ensino que sejam claros e possibilitem o planejamento de atividades para o desenvolvimento desses comportamentos.

O estudo de Viecili (2008) buscou identificar classes de comportamentos relacionadas à capacitação profissional do psicólogo para intervir por meio de pesquisa. Seu estudo foi feito com base nas Diretrizes Curriculares Nacionais para os Cursos de Graduação em Psicologia e em projetos de cursos de graduação de diferentes instituições de ensino do país. As classes de comportamentos identificadas e as lacunas presentes em algumas classes de comportamentos indicam, segundo a autora, que muitas classes de comportamentos relativas à capacitação científica do profissional ainda precisam ser descobertas, a fim de que colaborem com uma organização dos cursos de Psicologia de modo que garantam o desenvolvimento de comportamentos científicos fundamentais ao psicólogo.
Para completar e aperfeiçoar uma das classes de comportamentos caracterizadas por Viecili (2008), relacionada a delimitar problema de pesquisa, Noceti (2011) objetivou identificar os comportamentos constituintes da classe geral "delimitar problema de pesquisa a partir de perguntas" derivados de um manual de pesquisa. $\mathrm{O}$ autor destaca que "delimitar um problema" faz parte do processo de produção de conhecimento científico, sendo uma das etapas iniciais desse processo. Da mesma forma, "avaliar a confiabilidade de informações" (Luca, 2008) e "ler textos acadêmicos" (Goecks, 2011) constituem comportamentos acadêmicos de grande importância para a formação profissional e científica dos estudantes, pois com o desenvolvimento deles se tem a possibilidade de desenvolver outros comportamentos profissionais que serão necessários nas mais diferentes áreas de atuação. Além disso, é importante destacar que "ler textos acadêmicos" e "avaliar a confiabilidade de informações" são comportamentos requeridos ao longo do curso de graduação, bem como na pós-graduação e na atuação profissional. No entanto, além de não parecerem ser objetos de estudo como classes gerais de comportamentos, esses repertórios se apresentam frequentemente como insuficientes ou insatisfatórios pelos estudantes.

Viecili (2008) discute que quando um estudante aprende pesquisa como um conjunto de técnicas e procedimentos, é provável que não aprenda a identificar necessidades de intervenção e somente reproduza as técnicas aprendidas, conforme foi indicado por Ades (1981). Viecili ressalta ainda que a formação científica de qualidade precisa ser garantida na formação de psicólogos para possibilitar ampliação do campo de atuação profissional e atender as necessidades presentes na sociedade. Assim, a partir da caracterização das classes de comportamentos profissionais que compõem a formação científica do psicólogo é possível formular objetivos de ensino claros que permitam a elaboração de programas de ensino para desenvolver tais comportamentos. Estudos com o objetivo de caracterizar outras classes de comportamentos relacionadas à formação cientifica do psicólogo ou ao aperfeiçoamento e ampliação das classes já 
caracterizadas podem contribuir com um ensino mais eficaz de tais comportamentos, resultando em uma formação profissional que garanta que os estudantes sejam capacitados a intervir por meio de pesquisa.

\section{Características dos Objetivos de Ensino Presentes nos Planos de Curso e de Ensino de Disciplinas Relacionadas à Capacitação Científica na Graduação em Psicologia}

O estudo de Grando (2009) teve por objetivo caracterizar quais comportamentos científicos são propostos como objetivos de ensino de planos de curso e de disciplinas relacionadas à capacitação científica de estudantes de curso de graduação em Psicologia. A autora questiona em seu trabalho se os cursos de graduação em Psicologia estariam capacitando os futuros profissionais a produzirem conhecimento, de forma que esse fazer científico proporcionasse também que os psicólogos formados buscassem tecnologias e procedimentos mais eficazes e adequados para as diferentes demandas profissionais.

Após a identificação das características dos objetivos de ensino presentes nos planos de curso e nos planos de disciplinas relacionadas à capacitação científica do psicólogo, a autora as distribuiu em quatro categorias: atividades ou atuações escolares dos alunos; expressões vagas, genéricas ou ambíguas; declarações de intenção e atividades ou ações de professores. Com essa investigação, Grando (2009) concluiu que são poucos os professores que têm formulado objetivos de ensino de forma clara e que explicitem quais os comportamentos que o aluno deve aprender com as atividades da disciplina. Foi observado que, com frequência, os objetivos de ensino são apresentados em termos amplos, com a descrição do conteúdo das disciplinas ou da atividade que o professor realizará e não com a descrição do comportamento que se espera que o aluno seja capaz de apresentar. Desse modo, o trabalho de Grando (2009) traz como contribuição que o estudo das características dos objetivos de ensino, apresentados nos planos de curso e de ensino de disciplinas relacionadas à formação científica durante a graduação em Psi- cologia, pode tornar possível aos professores formularem planos com objetivos mais claros e precisos a respeito dos comportamentos-alvo a serem desenvolvidos. Assim, com uma consequente formação profissional e científica de melhor qualidade, os futuros profissionais estariam mais aptos a se tornarem autônomos e produzirem conhecimento científico, tanto como pesquisadores, como também em atividades de ensino e intervenção em diferentes áreas de atuação.

\section{Programas de Ensino de Comportamentos Profissionais que Compõem a Formação Científica do Psicólogo}

A presente categoria foi formulada com base no estudo desenvolvido por Luca (2013), em sua tese de doutorado. A partir da caracterização de componentes de comportamentos básicos constituintes da classe geral de comportamentos denominada "avaliar a confiabilidade de informações" realizada na dissertação de mestrado (Luca, 2008), o autor objetivou, em sua tese de doutorado avaliar qual o grau de eficiência e de eficácia de um programa de ensino para desenvolver classes de comportamentos constituintes dessa classe geral caracterizada.

A aplicação do Programa de Ensino da classe geral "avaliar a confiabilidade de informações", constituída por 866 classes de comportamentos, das quais 136 foram selecionadas para serem ensinadas, foi realizada com dois grupos: (a) Grupo História, composto por três aprendizes que cursavam graduação em História e 13 encontros para desenvolver os comportamentos caracterizados; e o (b) Grupo Psicologia, composto por quatro aprendizes do curso de graduação em Psicologia e 15 encontros. A avaliação da eficiência do Programa foi realizada pela avaliação do desempenho individual dos aprendizes em relação ao desenvolvimento de cada um dos comportamentos propostos no programa de ensino.

A avaliação da eficácia do programa de ensino foi feita por follow up realizado 5 meses após o término do Programa, em que foi aplicada outra atividade, e realizada entrevista a respeito das implicações e contribuições percebidas pelos aprendizes das aprendizagens desenvolvidas 
pelo programa em variados contextos. Foi verificado um alto grau de eficiência do programa de ensino no desenvolvimento dos comportamentos constituintes da classe geral "avaliar a confiabilidade de informações" e um alto grau de eficácia, evidenciado no follow up. $\mathrm{O}$ autor discute que a eficiência e a eficácia do Programa foram determinadas provavelmente pela forma de elaboração de tal programa, sendo realizado sob orientação dos princípios da Análise Experimental do Comportamento, o que contribuiria na garantia do desenvolvimento dos comportamentos.

Os aprendizes relataram uma série de implicações positivas produzidas pelas aprendizagens desenvolvidas no Programa (Luca, 2013), tais como: melhorar a qualidade do próprio discurso oral, melhorar a qualidade de trabalhos escritos, identificar e examinar informações preconceituosas, fundamentar melhor conclusões, apresentar argumentos completos e confiáveis em discursos, entre outras implicações. Tais relatos parecem demonstrar que o programa foi eficiente para o desenvolvimento de classes de comportamentos importantes para a atuação do profissional, destacando aqui a do psicólogo. E algumas das implicações relatadas não consistiam em comportamentos que foram objetivo do programa, mostrando que as aprendizagens desenvolvidas parecem ter dado base para que outros comportamentos fossem desenvolvidos também fora do contexto de aprendizagem, o que aponta para uma contribuição ainda maior desse tipo de programa na capacitação profissional e científica de estudantes. Vale destacar que várias dessas classes de comportamentos poderiam ser caracterizadas como parte da formação científica do psicólogo para atuação em qualquer campo ou âmbito profissional.

\section{Considerações Finais}

A partir da análise dos trabalhos selecionados, é possível identificar as contribuições desses estudos ao exame da importância e do papel da formação científica para a capacitação profissional do futuro psicólogo. Destaca-se ser unânime o papel fundamental da formação em pesquisa como parte necessária e indissociável para uma boa formação profissional. Fato esse que nos anos 1980 e 1990, com as discussões sobre a ausência de espaço para pesquisa no Currículo Mínimo, resultou nas Diretrizes Curriculares Nacionais para os cursos de graduação em Psicologia (Cruces, 2008). No entanto, apesar de garantir a existência do ensino de pesquisa na graduação do futuro psicólogo, não explicita de que forma essa formação ocorrerá, muitas vezes tornando-se apenas reprodução de técnicas e procedimentos dos conhecidos manuais de pesquisa (Viecili, 2008).

Parece que ainda é preciso avançar com relação à forma como é realizada a capacitação científica para que a pesquisa não seja vista de modo idealizado e desinteressante pelos estudantes. Muitos desses que, não tendo interesse em seguir a área acadêmica como docentes e pesquisadores, assumem uma postura de não realizadores de pesquisa, como se pesquisa fosse exclusivamente o que é feito formalmente dentro dos projetos de iniciação científica, laboratórios e práticas obrigatórias de disciplinas de metodologia científica e não como algo que também embasa uma prática profissional. É provável que isso se deva em boa parte pela falta de divulgação das oportunidades de experiências com pesquisa, fato esse para o qual se deve pensar em alternativas que incentivem os alunos a buscarem tais atividades (Bariani, 1995; Bastian et al., 2000).

Boa parte do desinteresse também está relacionada à falta de conhecimento sobre o quanto tal experiência vai, de fato, agregar ao desenvolvimento de comportamentos que serão úteis para a futura atuação profissional. $\mathrm{O}$ que parece indicar um desconhecimento acerca dos comportamentos que constituem o fazer científico pelos estudantes, psicólogos e até mesmo, por alguns docentes. Não parece difícil que uma formação em pesquisa desinteressante seja falha, sem relação com os ganhos que pode trazer ao profissional e que não faça sentido à realidade em que o aluno de graduação está inserido. Desse modo, além do importante questionamento acerca dos aspectos sociais, políticos e históricos por trás da condição atual da formação de nível superior, deve-se refletir sobre que tipo de mudanças 
práticas poderiam ser sugeridas como possíveis caminhos para uma melhoria da qualidade da formação profissional do psicólogo.

Talvez seja pertinente, também, pensar sobre quais comportamentos relativos ao fazer ciência podem ser desenvolvidos e quais seriam as condições para que isso acontecesse (Grando, 2009; Noceti, 2011; Viecili, 2008). Portanto, considerando que a formação científica é importante para a formação do futuro profissional de Psicologia, de que formas, atualmente, é possível e necessário ensinar pesquisa? É provável que a resposta a essa pergunta não aponte para um caminho apenas ou para alguma espécie de "fórmula" de ensino.

Os estudos com base na Programação de Ensino analisados neste artigo parecem trazer contribuições relevantes sobre esse questionamento ao apresentá-la como uma tecnologia eficaz para o entendimento e desenvolvimento de comportamentos científicos/profissionais (Goecks, 2011; Grando, 2009; Luca, 2008, 2013; Noceti, 2011 e Viecili, 2008). Novos estudos complementando a caracterização dos comportamentos apresentados e estudos a respeito da elaboração e avaliação de programas de ensino para desenvolvimento de outras classes gerais de comportamentos relacionadas à pesquisa podem configurar um caminho promissor na busca por uma capacitação eficaz e de qualidade. Destaca-se que a inclusão dos trabalhos realizados pelo grupo do PPGP da UFSC reflete que ainda existe pouca variedade de pesquisas nessa área, concentrando-se em pesquisas produzidas por esse grupo específico.

\section{Referências}

Ades, C. (1981). Treino em pesquisa, treino em compreensão. Psicologia: Ciência e Profissão, 1(1), 107-140.

Bariani, I. C. D. (1995). Um estudo exploratório sobre os motivos e os significados associados a prática da pesquisa em estudantes universitários. Estudos de Psicologia (Campinas), 12(1), 57-63.

Bastian, F. S., Amoretti, J., Rocha, K. B., Lisboa, C. S. de M., Kude, V. M. M., \& Wagner, A. (2000). Despertando o interesse pela pesquisa científica em psicologia. Psico, 31(1), 123-131.
Cruces, A. V. V. (2008). A pesquisa na formação de psicólogos brasileiros e suas políticas públicas. Boletim: Academia Paulista de Psicologia, 28(2), 240-255.

Decreto-lei no 53.464 de 21 de janeiro de 1964. (1964, 24 jan.). Diário Oficial da União.

Goecks, C. A. (2011). Comportamentos constituintes da classe geral de comportamentos "ler textos acadêmicos" (Dissertação de mestrado, Universidade Federal de Santa Catarina, Florianópolis, SC, Brasil). Recuperado em http://repositorio. ufsc.br/xmlui/handle/123456789/95870

Grando, A. P. (2009). Características dos objetivos de ensino presentes nos planos de curso e de ensino de disciplinas relacionadas à capacitação científica de alunos de cursos de graduação em psicologia (Dissertação de mestrado, Universidade Federal de Santa Catarina, Florianópolis, SC, Brasil). Recuperado em http://repositorio. ufsc.br/xmlui/handle/123456789/92843

Guareschi, N. M. de F., Wendt, G. W., \& Dhein, G. (2011). As atividades de pesquisa, extensão e monitoria na formação em psicologia. Avaliação Psicológica, 19(3), 387-403.

Kienen, N., Kubo, O. M., \& Botomé, S. P. (2013). Ensino programado e programação de condições para o desenvolvimento de comportamentos: Alguns aspectos no desenvolvimento de um campo de atuação do psicólogo. Acta Comportamentalia, 21(4), 481-494.

Leme, M. I. da S. (2001). Iniciação à pesquisa científica. Psicólogo inFormação, 5(5), 25-34.

Luca, G. G. (2008). Características de componentes de comportamentos básicos constituintes da classe geral de comportamentos denominada "avaliar a confiabilidade de informações" (Dissertação de mestrado, Universidade Federal de Santa Catarina, Florianópolis, SC, Brasil). Recuperado em https://repositorio.ufsc.br/ bitstream/handle/123456789/91008/262431. pdf? sequence $=1$

Luca, G. G. (2013). Avaliação da eficácia de um programa de contingências para desenvolver comportamentos constituintes da classe geral "avaliar a confiabilidade de informações" (Tese de doutorado, Universidade Federal de Santa Catarina, Florianópolis, SC, Brasil).

Ministério da Educação, Conselho Nacional de Educação/Câmara da Educação Superior (2011). Resolução $n^{0} 5$, aprovada em 15/03/2011, fixa as 
Diretrizes Curriculares Nacionais para os cursos de graduação em Psicologia, estabelecendo normas para o projeto pedagógico complementar para a Formação de Professores de Psicologia. Brasília, DF: Autor.

Noceti, R. V. (2011). Classes de comportamentos constituintes da classe geral "delimitar problema de pesquisa a partir de perguntas” (Dissertação de mestrado, Universidade Federal de Santa Catarina, Florianópolis, SC, Brasil). Recuperado em http://repositorio.ufsc.br/xmlui/ handle/123456789/95473.

Seidl-de-Moura, M. L., Bosco, E., Diniz, L. F., \& Santos, T. G. (1993). A pesquisa em psicologia e o aluno de graduação. Psicologia: Reflexão $e$ Crítica, 6(1-2), 17-38.

Silva, K. L. (2007). Comportamentos que constituem a classe geral delimitar problema de pesquisa no trabalho cientifico de alunos de pós-graduação em psicologia (Dissertação de mestrado, Universidade Federal de Santa Catarina, Florianópolis, SC, Brasil). Recuperado em http://repositorio.ufsc.br/xmlui/handle/123456789/89946
Viecili, J. (2008). Classes de comportamentos profissionais que compõem a formação do psicólogo para intervir por meio de pesquisa sobre fenômenos psicológicos, derivadas a partir das diretrizes curriculares nacionais para cursos de graduação em psicologia e da formação desse profissional (Tese de doutorado, Universidade Federal de Santa Catarina, Florianópolis, SC, Brasil). Recuperado em http://www.cfh.ufsc. br/ ppgp/Juliane\%20Viecili.pdf

Weber, S. (1985). Currículo mínimo e o espaço da pesquisa na formação do psicólogo. Psicologia Ciência e Profissão, 5(2), 11-13. doi:10.1590/ S1414-98931985000200004

Recebido: $17 / 11 / 2014$

$1^{a}$ revisão: $28 / 04 / 2015$ Aceite final: 07/05/2015 